Document downloaded from:

http://hdl.handle.net/10251/179843

This paper must be cited as:

Nazemosadat-Arsanjani, SB.; Gasulla Mestre, I. (2020). Tunable True-Time Delay Operation in A Dispersion-Engineered Few-Mode Fiber. IEEE. 203-206.

https://doi.org/10.23919/MWP48676.2020.9314392

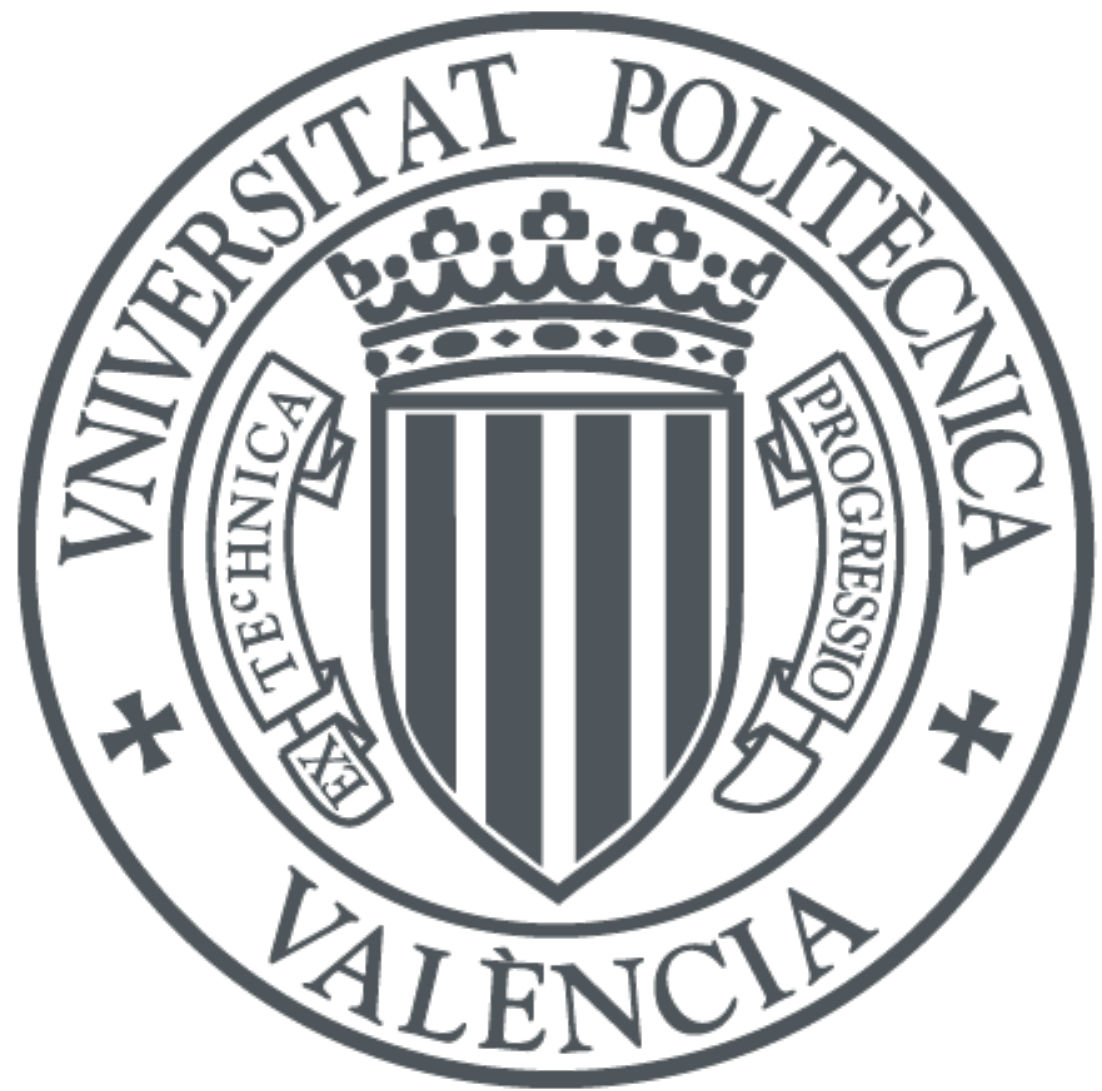

The final publication is available at

https://doi.org/10.23919/MWP48676.2020.9314392

Copyright IEEE

Additional Information 


\section{Tunable True-Time Delay Operation in A Dispersion-Engineered Few-Mode Fiber}

\author{
Elham Nazemosadat \\ ITEAM Research Institute \\ Universitat Politècnica de València \\ Valencia, Spain \\ sbnazars@iteam.upv.es
}

\author{
Ivana Gasulla \\ ITEAM Research Institute \\ Universitat Politècnica de València \\ Valencia, Spain \\ ivgames@iteam.upv.es
}

\begin{abstract}
We present a simple few-mode fiber design as a promising platform to implement tunable sampled true-time delay lines for radiofrequency signal processing. To the best of our knowledge, this is the first few-mode fiber ever reported featuring evenly spaced incremental dispersion values, which is an essential characteristic required for tunable operation of microwave photonics applications. The performance of the designed five-sample true-time delay line is theoretically validated in the context of microwave signal filtering, demonstrating free spectral range continuous tunability from $12.4 \mathrm{up}$ to $57 \mathrm{GHz}$.

Index Terms-Microwave photonics; Few-mode fiber; Optical delay lines; Optical signal processing.
\end{abstract}

\section{INTRODUCTION}

Space-division multiplexing over spatial modes of a fewmode fiber (FMF) has proven to be one of the promising solutions to the upcoming capacity crunch in conventional singlemode fiber networks [1]. Apart from optical transmission [2], FMFs have also been used in various signal processing applications, including few-mode optical parametric amplification [3], mode and wavelength conversion [4], multicasting [5], and realization of optical delay lines [6]-[9]. The latter case is particularly attractive for microwave photonics (MWP) applications, such as signal filtering, optical beam-steering in phased-array antennas and arbitrary waveform generation [10], [11], which operate based upon optical sampled true-time delay lines (TTDLs). The TTDLs provide a set of time-delayed replicas of an input radiofrequency (RF) signal that are evenlyspaced in time. MWP signal processing can benefit from the use of FMFs, given their ease of fabrication, compactness, low weight and performance versatility.

The implementation of a TTDL in a FMF is possible due to the fact that, in general, each spatial mode in a FMF features a different group delay and chromatic dispersion. Therefore, since the modes do not travel with the same group velocity, after propagating through the FMF, they can provide different time-delayed replicas of the input signal. If the FMF is designed such that the differential time delay between adjacent replicas is constant, then a TTDL can be realized. Furthermore, since the modes within one mode-group usually have similar dispersion characteristics, they are not suitable for providing the different time-delayed samples, and one should consider different mode-groups instead. The operating principle of a FMF-based TTDL is illustrated in Fig. 1, where the TTDL is fed by a single-carrier optical source and the spatial diversity of the FMF is used to provide the equallyspaced time-delayed replicas of the modulated input signal. If a multi-carrier optical source is used instead, wavelength diversity could be exploited in conjunction with the spatial diversity, leading to a 2-dimensional operation.

Even though some FMF-based TTDLs have been experimentally demonstrated to date, none of them are tunable with the operating wavelength, since either the delay line could only operate at a single wavelength [6]-[8], or the time delay had an insignificant dependence on the operating wavelength [9]. Recently, a tunable TTDL based on a ring-core FMF has been proposed [12]; however, it requires the inscription of several long period gratings to adjust the group delay and also chromatic dispersion of the modes, which adds to the complexity of the structure. In this work, we present a novel double-clad step-index FMF design, with tailored chromatic dispersion properties, that simply solves the aforementioned issues and operates as a continuously tunable TTDL for MWP applications.

\section{Principles of Few-Mode Fiber Tunable TRue-Time Delay LiNes}

In a FMF-based TTDL operating in the spatial diversity domain, the different samples are provided by the different modes propagating in the fiber (Fig. 1). For a given spatial mode $n$, the group delay, $\tau_{n}$, can be expanded in a 1-st order Taylor series around a center wavelength $\lambda_{0}$, as:

$$
\tau_{n}(\lambda)=\left[\tau_{0, n}+D_{0, n}\left(\lambda-\lambda_{0}\right)\right] L
$$

where $\tau_{0, n}$ and $D_{0, n}$ are the group delay per unit length and chromatic dispersion of mode $n$ at $\lambda_{0}$, respectively, while $L$ is the fiber length. The basic differential group delay (DGD) between modes $n$ and $n+1$ in the spatial diversity regime is then expressed as:

$$
\Delta \tau(\lambda)=\left[\Delta \tau_{0}+\Delta D\left(\lambda-\lambda_{0}\right)\right] L
$$

where $\Delta \tau_{0}=\tau_{0, n+1}-\tau_{0, n}$ and $\Delta D=D_{0, n+1}-D_{0, n}$ are the DGD and differential chromatic dispersion between adjacent modes at the center wavelength, respectively. For proper operation of a sampled TTDL at a given wavelength, it 


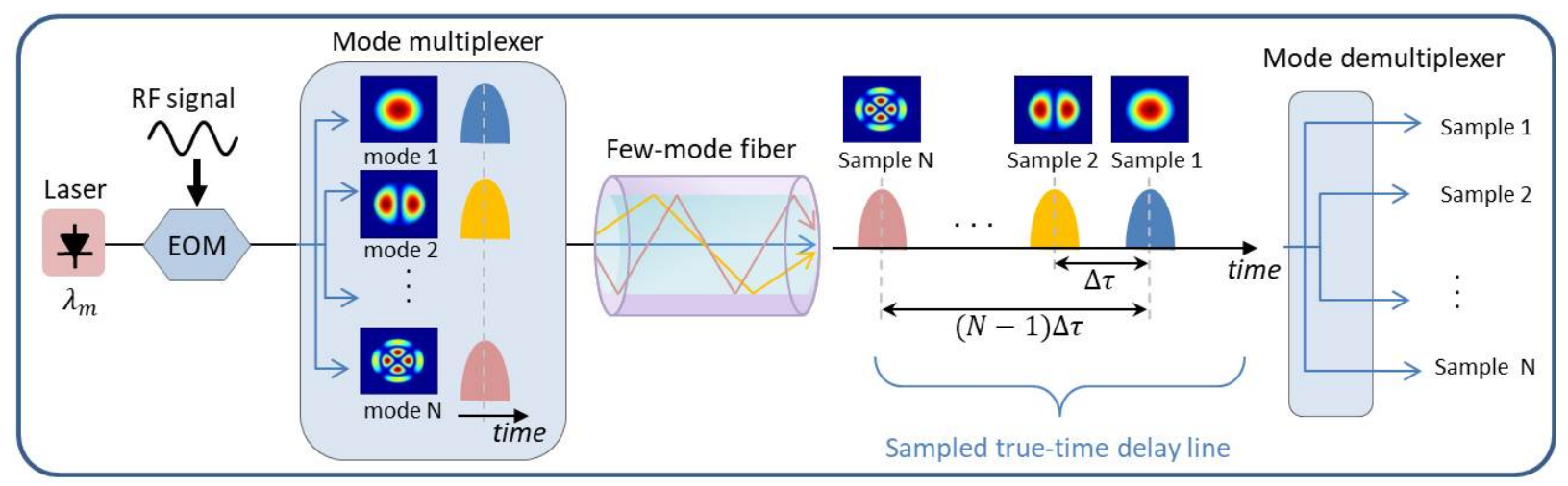

Fig. 1. Principle of operation of a FMF-based sampled delay line exploiting the spatial diversity of the fiber.

is essential to have a constant $\Delta \tau$ between adjacent samples at that wavelength. Furthermore, if $\Delta \tau$ changes linearly with the optical wavelength, a tunable delay line can be obtained. Based on (2), this means that a tunable FMF-based TTDL requires the satisfaction of two conditions, i.e., a constant differential group delay $\Delta \tau_{0}$ and a constant differential chromatic dispersion $\Delta D$, between adjacent modes at the center wavelength.

The FMF-based TTDLs reported so far do not exhibit a constant $\Delta D$ and therefore are not tunable with the wavelength [6]-[9], [13]. However, even with a constant $\Delta D$, there is still an issue that needs to be addressed in order to achieve tunable delay lines. In general, $\Delta \tau_{0}$ is several orders of magnitude larger than $\Delta D$ in FMFs, making the contribution of the second term in (2) negligible and thus leading to delay lines that are not suitable for MWP applications due to their low tunability. To solve this issue, we propose using external delay lines after the demultiplexer, at the fiber output, to adjust the DGD among the modes and decrease $\Delta \tau_{0}$ down to a value that is comparable to $\Delta D$. This way, both terms in (2) will contribute to the DGD in a similar manner and as a result a tunable delay line can be realized. Moreover, as well as reducing $\Delta \tau_{0}$ using the external delay lines, it should also be made sure that it is constant. This means that even if the original $\Delta \tau_{0}$ of the FMF is not constant, we can still fulfill the first requirement for tunable true-time delay operation since we are externally adjusting $\Delta \tau_{0}$. Thus, without having to be concerned about the first condition, we only have to design the FMF such that a constant and as large as possible $\Delta D$ is obtained.

\section{FMF DESIGN FOR TTDL OPERATION}

In addition to a constant and large $\Delta D$, the FMF should exhibit low mode coupling, low macro-bend losses and low nonlinearity. This means that the differential effective refractive index $\left(\Delta n_{\text {eff }}\right)$ between adjacent groups of modes, the relative effective refractive index difference of the modes with respect to the cladding $\left(n_{e f f}-n_{c l}\right)$ and the effective mode area of the modes $\left(A_{\text {eff }}\right)$ should all be large, respectively [14]. For the ease of fabrication, we have considered a FMF with a step-index core. However, in a conventional step-index FMF, $\Delta D$ between adjacent modes (or mode-groups) is not constant.
Therefore, an inner cladding has been added to the design to modify the dispersion properties of the modes in such a way that a constant $\Delta D$ is achieved. The refractive index profile of the designed double-clad step-index FMF is depicted in Fig. 2. The silica core is doped with $8 \mathrm{~mol} \% \mathrm{GeO} 2$ and has a radius of $a_{1}=9.1 \mu \mathrm{m}$, while the $\mathrm{GeO} 2$ concentration of the inner cladding is $5.96 \mathrm{~mol} \%$ and its radius is $a_{2}=13 \mu \mathrm{m}$. The outer cladding is pure silica with a radius of $62.5 \mu \mathrm{m}$. The effective refractive index of the supported modes in the first five modegroups are shown in Fig. 2. Among them, five modes $\left(\mathrm{LP}_{01}\right.$, $\mathrm{LP}_{11}, \mathrm{LP}_{21}, \mathrm{LP}_{31}$ and $\left.\mathrm{LP}_{41}\right)$, fulfill the conditions required to operate as a TTDL. The minimum $\Delta n_{\text {eff }}$ between these modes, which belong to different mode-groups, is $1.3 \times 10^{-3}$, which is comparable to that of typical commercial FMFs with a low-crosstalk [14]. The $\Delta n_{\text {eff }}$ among the modes within one mode-group is larger than $0.4 \times 10^{-3}$. The main properties of the five modes of interest are summarized in Table I. The large $A_{\text {eff }}$ of the modes significantly suppresses the intra and intermodal nonlinearities.

TABLE I

CHARACTERISTICS OF THE MODES OF INTEREST AT $1550 \mathrm{~nm}$

\begin{tabular}{c|ccccc} 
& $\mathrm{LP}_{01}$ & $\mathrm{LP}_{11}$ & $\mathrm{LP}_{21}$ & $\mathrm{LP}_{31}$ & $\mathrm{LP}_{41}$ \\
\hline$\left(n_{\text {eff }}-n_{c l}\right) \times 10^{-3}$ & 11.1 & 9.8 & 8.2 & 6.3 & 4.3 \\
$\mathrm{D}(\mathrm{ps} / \mathrm{nm} / \mathrm{km})$ & 20.47 & 22.25 & 24 & 25.77 & 27.56 \\
DGD vs. LP & & & & & \\
$A_{\text {eff }}\left(\mu m^{2}\right)$ & 213 & 314 & 356 & 393 & 425
\end{tabular}

The chromatic dispersion of the modes of interest, as a function of the wavelength, is demonstrated in Fig. 3. The inset depicts the average $\Delta D$ for different wavelengths (red dots). The corresponding error bars (standard deviation) of $\Delta D$ are also shown in the inset, which indicate how far the fiber is from being ideal (having a constant $\Delta D$ ). In the C-band, the maximum error is $2.6 \%$ at $1530 \mathrm{~nm}$. As will be seen later in Fig. 5, this amount of error is tolerable and still results in an acceptable response for MWP applications, such as signal filtering. 


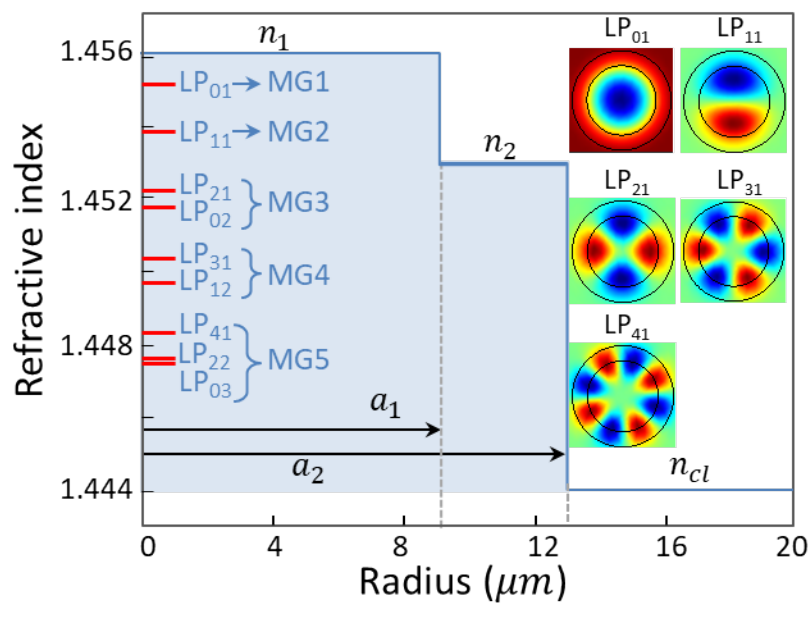

Fig. 2. Refractive index profile of the designed FMF. The effective refractive index of the supported modes in the first five mode-groups (MGs), at 1550 $\mathrm{nm}$, are shown in red. The transverse mode profiles of the modes of interest, which fulfill the conditions to operate as a FMF-based TTDL, are illustrated on the right.

Fig. 4(a) displays the DGD of the modes with respect to $\tau_{0,1}$, which is the group delay of $\mathrm{LP}_{01}$ at $\lambda_{0}=1520 \mathrm{~nm}$. The average $\Delta \tau$ is shown in the inset. As observed, $\Delta \tau_{0} \approx 2.35(\mathrm{~ns} / \mathrm{km})$, which is more than three orders of magnitude larger than $\Delta D \approx 1.78(\mathrm{ps} / \mathrm{nm} / \mathrm{km})$. Therefore, the corresponding RF processing range (given by the inverse of $\Delta \tau$ ) of a given microwave signal processing functionality based on such a TTDL would have a very limited tunability over the C-band. For the particular case of microwave signal filtering, the RF processing range corresponds to the Free Spectral Range (FSR). However, if we add suitable external delay lines after the demultiplexer at the output of the fiber,

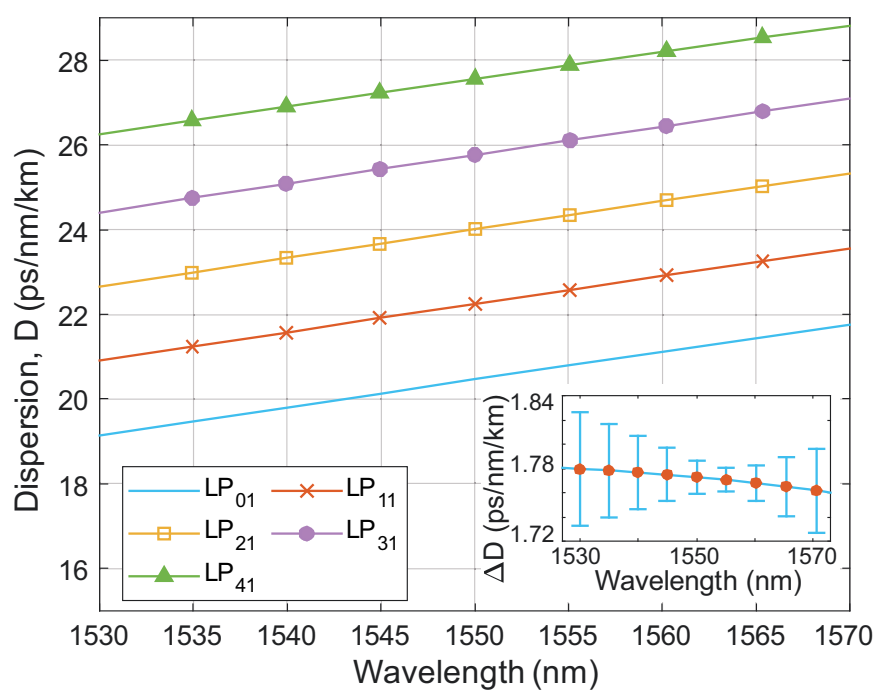

Fig. 3. Chromatic dispersion of the modes of interest. The inset shows the average $\Delta D$ and the associated error bars for different wavelengths. The maximum error in the C-band is $2.6 \%$ at $1530 \mathrm{~nm}$.

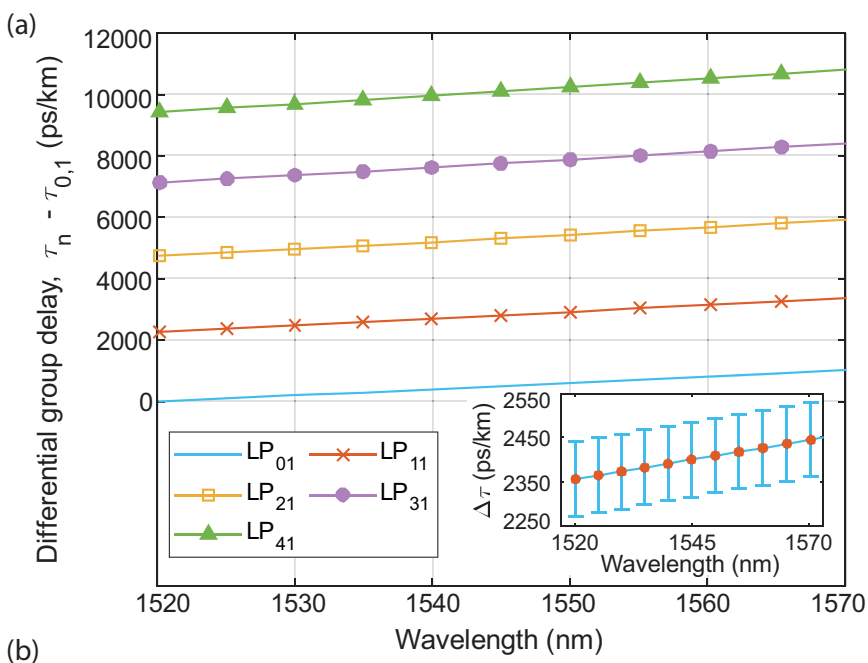

(b)

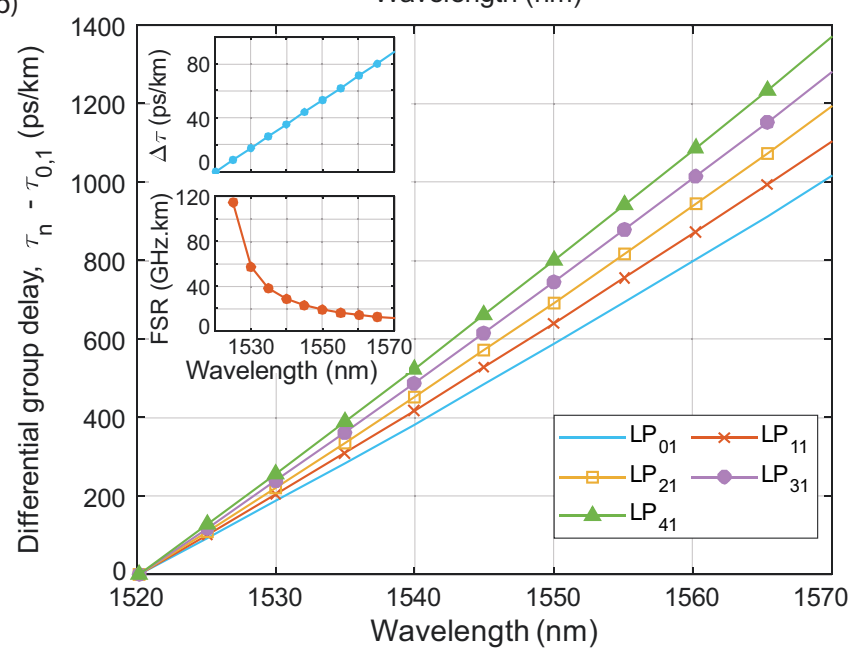

Fig. 4. Differential group delay per unit length between the TTDL samples with respect to the first sample carried by $\mathrm{LP}_{01}$, (a) before and (b) after employment of external delay lines. The average $\Delta \tau$ are shown in the insets for both cases. It can be observed that $\Delta \tau$ is significantly reduced when external delay lines are used (for example $\Delta \tau$ drops from $2.4 \mathrm{~ns} / \mathrm{km}$ to $53 \mathrm{ps} / \mathrm{km}$ at $1550 \mathrm{~nm}$ ). The corresponding free spectral range of a microwave filter implemented with a 1-km FMF is also shown in the bottom inset of (b).

we can significantly reduce $\Delta \tau_{0}$, without altering the required chromatic dispersion behaviour. The external delay can be adjusted such that at a given wavelength $(1520 \mathrm{~nm}$ in our case), $\Delta \tau_{0}=0$ is obtained, as shown in Fig. 4(b). Thereafter, since $\Delta D$ is constant, $\Delta \tau$ will change linearly with the optical wavelength and a tunable TTDL can be realized in the telecom optical wavelength bands. The top and bottom insets of Fig. 4(b) display the average $\Delta \tau$ and the corresponding FSR of a microwave filter realized by gathering together all the samples coming from the proposed TTDL and detecting them using a single photodetector, respectively. The performance of such a microwave filter can be observed in Fig. 5, where the calculated transfer function of the resulting 5-tap microwave filter is presented as a function of the RF frequency, for two different operation wavelengths. The FMF is considered 1-km long. For comparison, the filter response of an ideal FMF with 
(a)

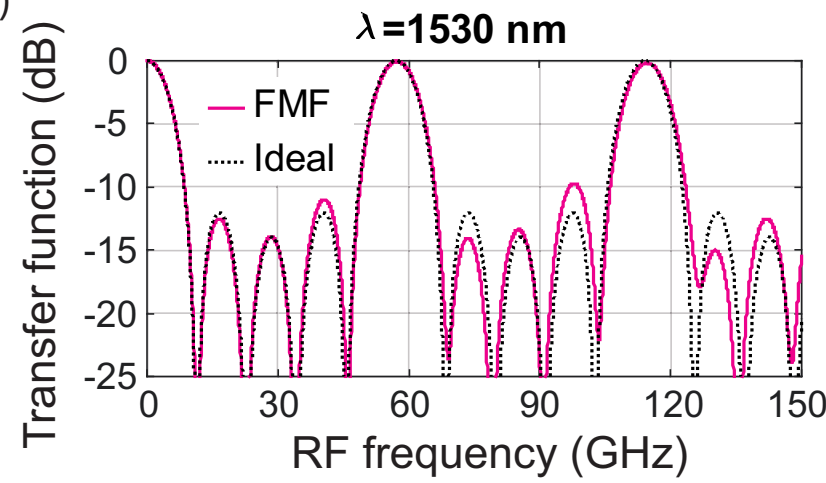

(b)

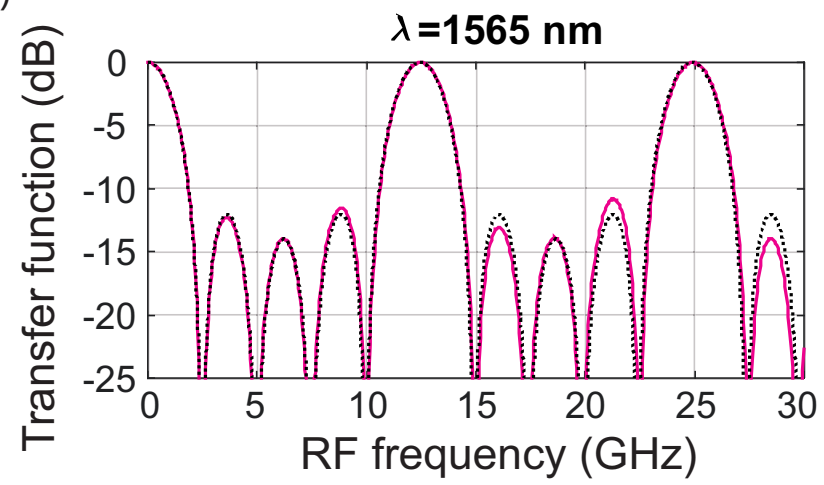

Fig. 5. Calculated transfer function of a microwave signal filter based on the proposed (solid line) and ideal (dashed line) TTDL for a 1-km FMF link while operating in the spatial diversity, at (a) $1530 \mathrm{~nm}$ and (b) $1565 \mathrm{~nm}$. Free Spectral Range continuous tunability from 12.4 up to $57 \mathrm{GHz}$ is obtained.

a constant $\Delta \tau$ is also shown. The designed FMF-based TTDL provides adequate results and is tunable over a broad range from 80.4 to 17.5 ps within the C-band, which corresponds to tuning the FSR of the filter continuously from $12.4 \mathrm{GHz}$ at $1565 \mathrm{~nm}$ to $57 \mathrm{GHz}$ at $1530 \mathrm{~nm}$, respectively.

\section{CONCLUSIONS}

MWP can benefit substantially from the increased compactness and performance versatility brought by the inherent parallelism of space-division multiplexing fibers. In particular, the implementation of reconfigurable distributed signal processing based on optical TTDL operation over a FMF requires continuous time delay tunability with the optical wavelength, which is usually insignificant within the frequency ranges that are suitable for MWP applications. This is due to the inherent propagation characteristics of FMF modes. In this work, we propose a feasible and simple method for solving this limitation by designing a novel double-clad step-index FMF, in which the chromatic dispersion among adjacent groups of modes increases in constant steps, along with the inclusion of external delay lines at the FMF output to modify the DGD among the modes. Wide continuous tunability of a fivesampled TTDL is demonstrated in a 35-nm wavelength range, which in a microwave signal filtering scenario translates into FSR tunability from 12.4 to $57 \mathrm{GHz}$ for a 1-km FMF link. This approach can be extended to perform additional microwave signal processing applications such as optical beamforming networks for phased array antennas or arbitrary waveform generation.

\section{ACKNOWLEDGMENT}

This work is supported by the European Research Council (ERC) under Consolidator Grant Project 724663, and Ramon y Cajal fellowship RYC-2014-16247 for I. Gasulla.

\section{REFERENCES}

[1] D. J. Richardson, J. M. Fini, and L. E. Nelson, "Space-division multiplexing in optical fibres", Nature Photonics, vol. 7, no. 5, pp. 354-362, 2013.

[2] R. Ryf, S. Randel, A. H. Gnauck, C. Bolle, A. Sierra, S. Mumtaz, M. Esmaeelpour, E. C. Burrows, R.-J. Essiambre, P. J. Winzer, D. W. Peckham, A. H. McCurdy, and R. Lingle, "Mode-division multiplexing over $96 \mathrm{~km}$ of few-mode fiber using coherent $6 \times 6$ mimo processing," J. Lightwave Technol., vol. 30, no. 4, pp. 521-531, Feb 2012.

[3] E. Nazemosadat, A. Lorences-Riesgo, M. Karlsson, and P. A. Andrekson, "Design of highly nonlinear few-mode fiber for C-band optical parametric amplification," Journal of Lightwave Technology, vol. 35, no. 14, pp. 2810-2817, 2017.

[4] R. J. Essiambre, M. A. Mestre, R. Ryf, A. H. Gnauck, R. W. Tkach, A. R. Chraplyvy, Y. Sun, X. Jiang, and R. Lingle, "Experimental investigation of inter-modal four-wave mixing in few-mode fibers," IEEE Photon. Technol. Lett., vol. 25, no. 6, pp. 539-542, March 2013.

[5] J. Gao, E. Nazemosadat, C. Yang, S. Fu, M. Tang, W. Tong, J. Carpenter, J. Schröder, M. Karlsson, and P. A. Andrekson, "Design, fabrication, and characterization of a highly nonlinear few-mode fiber", Photonics Research, vol. 7, no. 11, pp. 1354-1362, 2019.

[6] D. V. Nickel, C. Villarruel, K. Koo, F. Bucholtz, and B. Haas, "Few mode fiber-based microwave photonic finite impulse response filters," Journal of Lightwave Technology, vol. 35, no. 23, pp. 5230-5236, 2017.

[7] R. Guillem, S. García, J. Madrigal, D. Barrera, and I. Gasulla, "Fewmode fiber true time delay lines for distributed radiofrequency signal processing," Optics Express, vol. 26, no. 20, pp. 25761-25768, Oct 2018.

[8] S. García, R. Guillem, J. Madrigal, D. Barrera, S. Sales, and I. Gasulla, "Sampled true time delay line operation by inscription of long period gratings in few-mode fibers," Optics Express, vol. 27, no. 16, pp. 22787 22793, 2019.

[9] K. H. Lee, W. Y. Choi, S. Choi, and K. Oh, "A novel tunable fiber-optic microwave filter using multimode DCF," IEEE Photonics Technology Letters, vol. 15, no. 7, pp. 969-971, 2003.

[10] J. Yao, "Microwave Photonics," Journal of Lightwave Technology, vol 27, no. 3, pp. 314-335, Feb 2009.

[11] J. Capmany, J. Mora, I. Gasulla, J. Sancho, J. Lloret, and S. Sales, "Microwave photonic signal processing," Journal of Lightwave Technology, vol. 31, no. 4, pp. 571-586, 2013.

[12] S. García, R. Guillem, and I. Gasulla, "Ring-core few-mode fiber for tunable true time delay line operation," Opt. Express, vol. 27, no. 22 pp. 31773-31782, Oct 2019.

[13] J. Zhao, H. Zhang, Z. Yang, J. Xu, T. Xu and C. Wang, "Few-mode fibers with uniform differential mode group delay for microwave photonic signal processing," IEEE Access, vol. 8, pp. 135176-135183, 2020.

[14] P. Sillard, M. Bigot-Astruc, D. Boivin, H. Maerten, and L. Provost, "Few-mode fiber for uncoupled mode-division multiplexing transmissions," in 2011 37th European Conference and Exhibition on Optical Communication, Geneva, 2011, pp. 1-3. 